\title{
Colonic interposition for recurrent Gastroesophageal junction adenocarcinoma of stomach: A case report
}

\author{
Sujan Regmee, Shrijan Kharel, Tanka Prasad Bohara, Mukund Raj Joshi
}

\section{Abstract}

Gastroesophageal (GE) junction tumor is a common cancer and despite the advances in its surgical and medical management, recurrence is still seen. Esophagogastrectomy with colonic interposition may be required in case of recurrence, especially when the stomach is not suitable or available for tubularization and anastomosis. Here we report a 30 -yearold female who had undergone proximal partial gastrectomy 14 months ago followed by six cycle of chemotherapy for poorly differentiated mucinous adenocarcinoma of stomach. The patient was admitted with a 20 days history of dysphagia. A recurrence of the tumor was confirmed and a transhiatal esophagogastrectomy with interposition of right colon with terminal ileum with end to end anastomosis of oesophagus and ileal ends followed by colojejunostomy and ileocolostomy was done. All the resected margins were free of tumor with two out of four lymph nodes involved. Postoperative period was uneventful up to five months of surgery.

Keywords: Colonic transposition; Recurrent GE junction tumor; Transhiatal oesophagectomy.

\section{Author affiliations:}

Department of surgery,

Kathmandu Medical College Teaching Hospital

Correspondence:

Dr Sujan Regmee,

Kathmandu Medical College, Sinamangal Kathmandu, Nepal.

Email: sujanregmee@hotmail.com,

ORCID: https://orcid.org/0000-0002-73556531

\section{Copyright information:}

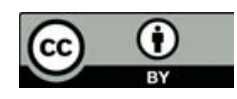

How to cite this article:

Regmee S, Kharel S, Bohara TP, Joshi MR. Colonic interposition for recurrent gastroesophageal junction adenocarcinoma of stomach: a case report. J Soc Surg Nep. 2020 Dec;23(2):63-66.

DOI:

https://doi.org/10.3126/jssn.v23i2.35843

\section{Introduction}

Gastric cancer is a major cause of cancer death worldwide. ${ }^{1}$ Although the potential curative treatment for gastric cancer is radical gastrectomy, early detection, improvements in extended lymphadenectomy, and adjuvant chemotherapy have all drastically improved the survival rate of gastric cancer. However, despite these advances, recurrence is still possible and is often the cause of unsuccessful recovery from gastric cancer. ${ }^{2,3}$ The reasons for local recurrence could be many like inadequate margin at the time of resection, biology of the disease, bad patient compliance.

Local recurrence may occur in patients who underwent R0 resection. Four types of local recurrence can be differentiated according to location and origin: 1) local lymph node metastasis, 2) extraluminal recurrence, 3) recurrence within the remnant stomach, and 4) anastomotic recurrence following gastrectomy. Surgical intervention has been limited to locoregional recurrent gastric cancer. ${ }^{4}$ Failure to appreciate the fact that the microscopic boundaries of the invading process may extend well beyond the palpable edge of the main lesion to involve distal esophagus in case of adenocarcinoma of the stomach during total or proximal subtotal gastrectomy leads to transection of the esophagus in an area invaded by disease, which predisposes to esophageal anastomotic recurrence. ${ }^{5,6}$ Positive margins were avoided only with resection of $12 \mathrm{~cm}$ or more of macroscopically tumor-free esophagus above the primary and negative gastric margin can be achieved by dividing the stomach 4 to $6 \mathrm{~cm}$ from palpable tumor. ${ }^{7,8}$

Esophagogastrectomy with colonic interposition may be required in case of distal esophageal carcinoma, carcinoma of proximal stomach and also in esophagogastric 
anastomotic recurrence, especially when the stomach is not suitable or available. Colon interposition, although often well tolerated, can sometimes be associated with restenosis, polyps or rarely adenocarcinoma. The cases with colonic transposition had an overall mortality and survival similar to those for gastric transposition. Therefore, it is usually a source of tissue for esophageal replacement whenever stomach is not available or suitable., ${ }^{9,10}$

\section{Case Report}

A 30-year-old female, who had undergone proximal partial gastrectomy about 14 months back for poorly differentiated mucinous adenocarcinoma of stomach, was admitted with a 20 days history of dysphagia which was initially for solid food but later progressed causing difficulty in swallowing liquid as well. In past 14 months patient had undergone 6 cycles of chemotherapy. On examination the general condition of the patient was not fair, she was cachectic and nutritional status was poor. Mildly distended upper abdomen was noticed. Upper GI endoscopy (Figure 1) revealed stricture at the site of the anastomosis through which endoscope could not be negotiated.

Reverse Transcriptase Polymerase Chain Reaction (RTPCR) for COVID-19 was sent along with other preoperative investigations as the patient had traveled from a COVID prevalent area and was planned for surgery during the pandemic. Positron Emitted Tomography (PET) scan was planned but could not be done due to unavailability of radioactive dye due to the pandemic.

A whole-body Magnetic Resonance Imaging (MRI) (Figure 2a and 2b) was done, which revealed circumferential thickening of the wall of lower esophagus adjacent to the anastomosis with restricted diffusion suggesting recurrence, although there was no evidence of distance metastasis.

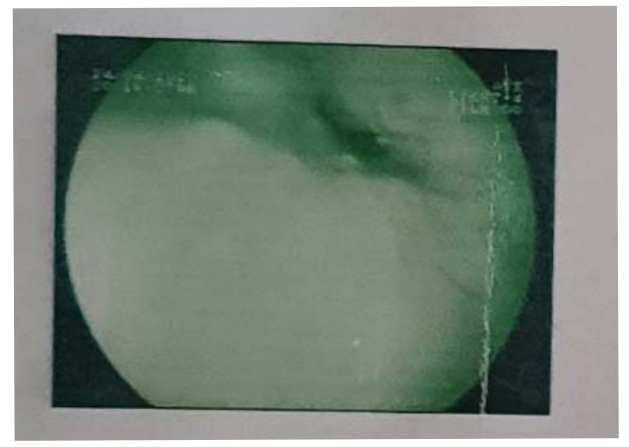

Figure 1. Endoscopic picture

A hard mass on neotubularized stomach and esophagus with proximal extent of $5 \mathrm{~cm}$ from previous anastomosis and distal extent up to antrum of stomach was seen intraoperatively (Figure 3).

Transhiatal esophagogastrectomy with interposition of right colon with terminal ileum with end to end

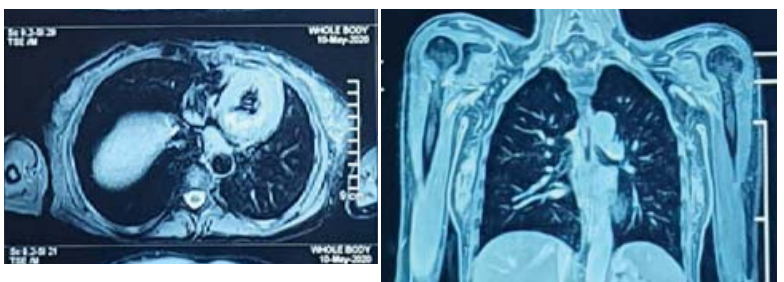

Figure 2a. Transverse View Figure 2b. Coronal View

anastomosis of oesophagus and ileal ends followed by colojejunostomy and ileocolostomy with feeding jejunostomy (FJ) placement with cholecystectomy (for Gall bladder calculi) with appendectomy was done. Biopsy of nodes from falciform ligament, pancreatic capsule were sent for histopathology.

Feeding was gradually increased via FJ starting from first postoperative day. On the second postoperative day, patient developed $\mathrm{B} / \mathrm{L}$ pleural effusion mild on right and moderate on left side for which left sided tube thoracostomy was done. X-Ray of chest with gastrograffin swallow was performed on 4th postoperative day which showed no leakage from the site of anastomosis. Oral feed was then started and gradually increased. Other parameter in the postoperative period was uneventful. Postoperative Computed Tomography (CT) scan of chest and abdomen (Fig. 4) was done on the 13th post-operative day, which showed no evidence of leakage of contrast at anastomotic site.

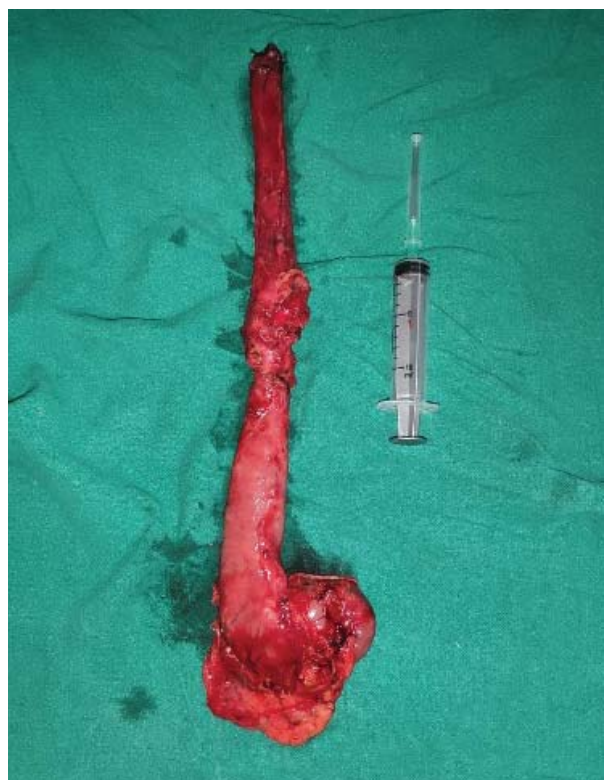

Figure 3. Resected specimen

The postoperative histopathology report showed a $3 \times 1.5$ $\mathrm{cm}$ tumor, recurrent poorly differentiated adenocarcinoma extending up to the adventitia. All the resected margin were free of tumor and two out of four lymph node were involved. Perineural and lymphovascular invasion of the tumor was seen. Postoperative period was uneventful up to five months of surgery. 


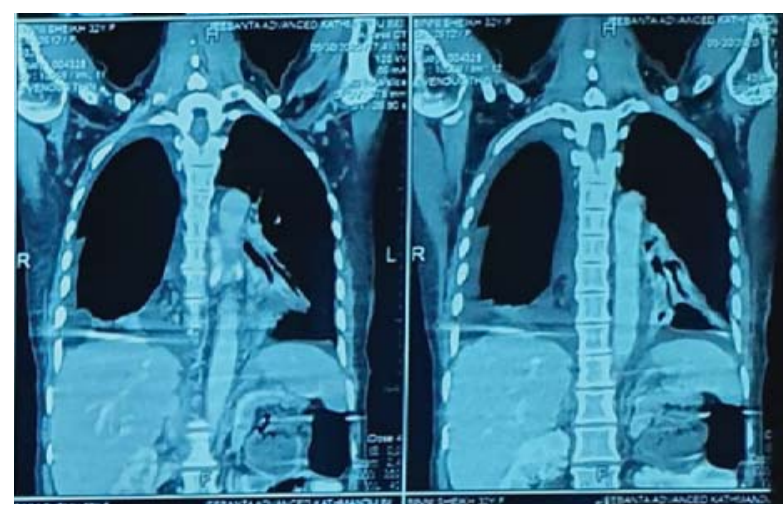

Figure 4. Postoperative CT scan of Chest and Abdomen

\section{Discussion}

Esophageal cancer has the tendency to spread intramurally through the submucosal lymphatics. The tumors of the GE junction or proximal stomach spreads both proximal up the esophagus and distal down into the stomach. Adequate esophageal and distal gastric margin is essential for the management of such tumors.

During surgery with a curative intent transection of the esophagus $4 \mathrm{~cm}$ above the main lesion resulted in a 70 percent incidence of positive margins and with a margin of $6 \mathrm{~cm}$ one may expect a 56 percent incidence of positive margins. ${ }^{11}$ Margins greater than $6 \mathrm{~cm}$ were considered adequate in a series; however, since fresh esophageal specimens shrink to half of their actual size after resection, the avoidance of positive esophageal margin will require resection of $12 \mathrm{~cm}$ of macroscopically tumor-free esophagus above the palpable edge of the primary lesion in those patients. The distal margin that is free of cancer on final histology examination can be achieved by dividing the stomach at least $4 \mathrm{~cm}$ from palpable tumor. ${ }^{8}$

When the lymph node dissection is considered according to American Joint Committee on Cancer (AJCC) guidelines for primary gastric cancer 15 nodes needs to be resected. For esophageal cancer a better survival can be expected in patients with a lymph node yield of $21 .{ }^{12}$ A mediastinal lymph node dissection is warranted in patients with a positive lymph node yield ranging from one to eight. ${ }^{13}$

The surgical reconstruction is the next factor that needs to be considered while operating on such patients. When enough stomach remains it can be used as an esophageal replacement. It is considered an ideal replacement as it has a rich vasculature in the sub mucosal plane, ease of mobilization and a single anastomosis is sufficient for reconstruction. ${ }^{14}$ However, when the stomach cannot be used due to more extensive primary disease or like in this case, needs to be resected due to recurrence of the disease, the jejunum or the interposed colon can to be used for esophageal reconstruction.

The jejunum can be used as a free flap interposition between the esophagus and the stomach. The basic advantage of jejunum is that it is an abundant organ with long length available for reconstruction. The jejunum has its own intrinsic peristalsis and its diameter is similar to that of the esophagus. ${ }^{15}$

Performing esophageal reconstruction with a segment of the colon provides good long-term function. Both the left and the right colon can be used for reconstruction. The left colon has an adequate blood drainage through the left colic and middle colic vein, its intrinsic mucus production also helps mitigate the acid reflux, and its isoperistaltic nature. However the need for three anastomosis is a challenge with this option. ${ }^{16}$

The right colon similarly provides a good option for reconstruction, the main issue with this option is that a marginal artery is absent in the right colon so the ileocecal arterial arch needs to be preserved, obtaining a segment of ileum for reconstruction. Moreover the peristalsis of this part of colon is also slower. ${ }^{17}$ The distal ileum segment was used for reconstruction in our case after considering all these factors. The ileal caliber seemed more appropriate for reconstruction during surgery.

In cases where enough stomach margin is not available, colon is the preferred source of tissue for esophageal replacement because the overall mortality and survival is similar to those for gastric transposition., 9,10

Complete removal of the primary tumor with an adequate margin of resection is the general goal of any oncologic procedure. Numerous studies demonstrate that the incidence of positive esophageal margins and anastomotic recurrence is high when the primary neoplasm is located high in the stomach and when the surgeon fails to resect enough esophagus. Size of the tumor, lymph node involvement, perineural and lymphovascular invasion and a poorly differentiated tumor are the factors associated with recurrence of GE junction tumor. ${ }^{18}$ In the first two years of surgery for a primary esophagogastric junction tumor, recurrence is observed in $20 \%$ to $50 \%$ of cases. ${ }^{19}$ The role of surgical resection in cases with recurrence is dubious, some report that it adds to survival while other report it does not. ${ }^{20}$

Badgwell in 2009 reported in his series of 60 patients that the surgical reintervention in patients with recurrence of GE junction and gastric cancer had a possible resection with curative intent in $29(52 \%)$ patients and the overall survival was $72 \%, 38 \%$ and $28 \%$ respectively at one, three and five years. ${ }^{19}$ The overall survival in this series was reported be high patients who had a resectable disease.

The late complications of interposed colon have been reported to include anastomotic stricture, diverticulosis, colitis, ulceration and cancer. It is a complex procedure that has increased morbidity, compared with gastric transposition. 


\section{Conclusion}

Transhiatal esophagogastrectomy with interposition of right colon is feasible in reccurent gastroesohageal junction cancer in highly selected patients.

\section{References}

1. Jemal A, Bray F, Center MM, Ferlay J, Ward E, Forman D. Global cancer statistics. CA Cancer J Clin. Mar-Apr 2011;61(2):69-90.

2. Kim JP, Kwon OJ, Oh ST, Yang HK. Results of surgery on 6589 gastric cancer patients and immunochemosurgery as the best treatment of advanced gastric cancer. Ann Surg. 1992 Sep;216(3):269-78.

3. Inokuchi K. Prolonged survival of stomach cancer patients after extensive surgery and adjuvant treatment: an overview of the Japanese experience. Semin Surg Oncol. Nov-Dec 1991;7(6):333-8.

4. Shchepotin I, Evans SR, Shabahang M, Cherny V, Buras RR, Zadorozhny A, et al. Radical treatment of locally recurrent gastric cancer. 1995 Apr;61(4):3716.

5. Paulino F, Roselli A. Carcinoma of the stomach with special reference to total gastrectomy. Curr Probl Surg. 1973 Nov;3-72.

6. Humphrey EW, Kersten TE. Adenocarcinoma at the esophagogastric junction. In: Varco RL, Delaney JP. Controversy in Surgery. Philadelphia and London: WB Saunders, 1976: 603-8.

7. Papachristou DN, Agnanti N, D'Agostino H, Fortner JG. Histologically Positive Esophageal Margin in the Surgical Treatment of Gastric Cancer. Am J Surg. 1980 May;139(5):711-3.

8. Paul D DiMusto, Mark B Orringer. Transhiatal Esophagectomy for Distal and Cardia Cancers: Implications of a Positive Gastric Margin. Ann Thorac Surg. 2007 Jun;83(6):1993-8.

9. Taslimi R, Jowkar A, Ghavam MRH, Tavasol T, Allameh SF, Rakhshani N. Squamous cell carcinoma (SCC) arising in esophageal colon interposition. Oxf Med Case Reports. 2017 Dec 18;2017(12):omx068.

10. Davis PA, Law S, Wong J. Colonic Interposition After Esophagectomy for Cancer. Arch Surg. 2003 Mar;138(3):303-8.

11. Sefton GK, Cooper DJ, Giddings A E, Grech P. Assessment and resection of carcinoma at the gastroesophageal junction. Surg Gynecol Obstet. 1977 Apr;144(4):563-6.

12. Sanchuan L, Tingting S, Xingkang H, Zhenghua L, Shujie C: Prognostic value of resected lymph nodes numbers for Siewert II gastroesophageal junction cancer. Oncotarget. 2017 Dec 20;9(2):2797-2809.

13. Mazer LM, Poultsides GA: What is the best operation for proximal gastric cancer and distal esophageal cancer? Surg Clin North Am. 2019 Jun;99(3):457469.

14. Buunen M, Rooijens PP, Smaal HJ, Kleinrensink GJ, Van Der Harst E, Tilanus HW, et al: Vascular anatomy of the stomach related to gastric tube construction. Dis Esophagus. Dis Esophagus. 2008;21(3):272-4.

15. Blackmon SH, Correa AM, Skoracki R, Chevray PM, Kim PM, Mehran RJ. et al. Supercharged pedicled jejunal interposition for esophageal replacement: a 10-year experience. Ann Thorac Surg. 2012 Oct;94(4):1104-11.

16. Wilkings EW Jr: Long-segment colon substitution for the esophagus. Ann Surg. 1980 Dec;192(6):7225 .

17. Matsumoto H, Hirai T, Kubota H, Murakami H, Higashida M, Hirabayashi Y: Safe esophageal reconstruction by ileocolic interposition. Dis Esophagus. 2012 Apr;25(3):195-200.

18. Neri A, Marrelli D, Voglino C, Mare GD, Ferrara $\mathrm{F}$, Marini $\mathrm{M}$, et al. Recurrence after surgery in esophago-gastric junction adenocarcinoma: current management and future perspectives. Surg Oncol. 2016 Dec;25(4):355-363.

19. Badgwell B, Cormier JN, Xing Y, Yao J, Bose D, Krishnan S, et al. Attempted salvage resection for recurrent gastric or gastroesophageal cancer. Ann Surg Oncol. 2009 Jan;16(1):42-50.

20. Carboni F, Lepiane P, Santoro R, Lorusso R, Mancini P, Carlini M, et al. Treatment for isolated loco-regional recurrence of gastric adenocarcinoma: does surgery play a role?. World J Gastroenterol. 2005 Nov 28;11(44):7014-7. 